\title{
Reduction of matrices over simple Ore domains
}

\author{
Victor Bovdi, Bohdan Zabavsky
}

Corresponding author: V. Bovdi

Address: UAEU, Al Ain, United Arab Emirates (V. Bovdi)

Ivan Franko National University, Lviv, Ukraine (B. Zabavsky)

\begin{abstract}
We study the theory of diagonal reductions of matrices over simple Ore domains of finite stable range. We cover the cases of 2 -simple rings of stable range 1, Ore domains and certain cases of Bezout domains.
\end{abstract}

Keywords and phrases: Ore domain, Bézout domain, stable range, $n$-simple ring, diagonal reduction of a matrix.

Mathematics Subject Classification: 19B10, 16E50, 16U10, 16U20

\section{Introduction and results}

The problem of diagonalization of matrices over rings is a classical problem of ring theory. An overview can be found in [9]. While commutative elementary divisor rings have been investigated fairly systematically, noncommutative elementary divisor rings have not received such attention. Nevertheless significant results have been obtained in this field. For example, Henriksen [6, Theorem 3, p. 134] showed that any matrix over an unit-regular ring can be reduced to a diagonal form by multiplications from left and right by invertible matrices of suitable sizes.

According to Cohn [4, Theorem 3.6, p.255], a right principal Bézout domain has the reduction matrix property, at least when certain conditions on the diagonal elements of its diagonal form are satisfied. An example of such Bézout domain was constructed in [5, Lemma, p. 27]; is should be noted that it is also an example for simple Bézout domains, i.e. domains with trivial two-sided ideals only.

The study of the connections between the stable range of a ring and the reduction of matrices over that ring showed (see for example [9, Theorem 4.4.1, p. 185]) that a simple Bézout domain was an elementary divisor ring if and only if it was a 2-simple domain. 
The notion of a stable range of a ring was introduced in algebraic $K$ theory and has been proved useful for the study of certain problems in the ring theory. In particular, it was proved that the stable range of an elementary divisor ring did not exceed 2 [9, Theorem 1.2.40, p.48] and each Bézout domain is a Hermite ring [1]. Several important results about connections between Bézout domains, Hermite rings, stable range and elementary divisor rings were obtain in the papers of Amitsur, Ara, Goodearl, Menal, Moncasi, O'Meara, Paphael and others (see for example [1, 2, 7, 9]). In the present paper we study the diagonal reduction of matrices over a simple Ore domain. This investigation reveals a connection to the theory of full matrices over certain classes of rings.

Our main results are the following.

Theorem 1. Let $R$ be a 2-simple ring of stable range 1 and let $a, b \in R$ be such that either $a b \neq 0$ or $b a \neq 0$. The matrix $\operatorname{diag}(a, b) \in R^{2 \times 2}$ can be reduced to the form $\operatorname{diag}(1, c) \in R^{2 \times 2}$ for some $c \in R$.

In the case of $(n+1)$-simple domains (where $n \geq 2$ ) we have the following.

Theorem 2. Let $R$ be an $(n+1)$-simple Ore domain of stable range $n \in \mathbb{N}$. For each non-zero divisor $A \in R^{(n+1) \times(n+1)}$ there exist $P, Q \in G L_{n+1}(R)$ and $A_{0} \in R^{n \times n}$ such that

$$
P A Q=\left(\begin{array}{ccc}
1 & 0 & \ldots \\
0 & 0 \\
\vdots & A_{0} \\
0 & &
\end{array}\right) .
$$

As a consequence of Theorem 2 we have the following result.

Theorem 3. Let $R$ be a 2-simple Ore domain of stable range 1. For each non-zero divisor matrix $A \in R^{2 \times 2}$ there exist $P, Q \in G L_{2}(R)$ such that

$$
P A Q=\left(\begin{array}{ll}
1 & 0 \\
0 & a
\end{array}\right) \quad \text { for some } a \in R
$$

Since each Bézout domain is an Ore domain [9, Corollary 2.1.1, p. 53] and [7], from Theorem 2 we have the following.

Theorem 4. Let $R$ be a n-simple Bézout domain. If $m \geq n \in \mathbb{N}$, then for each $A \in R^{m \times m}$ there exist $P, Q \in G L_{m}(R)$ such that $P A Q=\left(\begin{array}{cc}E & 0 \\ 0 & T \\ 0 & 0\end{array}\right)$, where $E$ is the identity matrix, $T=\operatorname{diag}\left(A_{1}, \ldots, A_{k}\right)$ and each $A_{i} \in R^{n \times n}$ is a triangular matrix. 


\section{Notations and Preliminary Results}

The set of positive integers is denoted by $\mathbb{N}$. Let $R$ be an associative ring with nonzero unit and let $r, s, n \in \mathbb{N}$. The vector space of matrices over the ring $R$ of size $r \times s$ is denoted by $R^{r \times s}$. Groups of units of the rings $R$ and $R^{n \times n}$ are denoted by $U(R)$ and $G L_{n}(R)$, respectively.

A ring $R$ is called right (left) Bézout ring if each finitely generated right (left) ideal of $R$ is principal. A ring $R$ which is simultaneously right and left Bézout ring is called Bézout ring. A domain $R$ is called right (left) Ore domain if for each $a, b \in R \backslash\{0\}$ one has $a R \cap b R \neq\{0\}(R a \cap R b \neq\{0\})$. Each Ore domain is a domain that is simultaneously right and left Ore domain and each Bézout domain is an Ore domain [8, Proposition 1.8, p. 53].

Each Ore domain $R$ can be embedded into a division ring 4, Proposition 5.2 , p. 259], so we can define ranks of a matrix $A$ over $R$ on their rows $\rho_{r}(A)$ and their columns $\rho_{c}(A)$, respectively. Note that, the numbers $\rho_{r}(A)$ and $\rho_{c}(A)$ do not change under elementary transformations of $A$.

The smallest $m \in \mathbb{N}$ such that a matrix $A \in R^{r \times s}$ is a product of two matrices of size $r \times m$ and $m \times s$, is called the inner rank $\rho(A)$ of $A[3$, p. 244]). Note that $\rho(A) \leq \min \left\{\rho_{r}(A), \rho_{c}(A)\right\}$ and the number $\rho(A)$ does not change under elementary transformations (see [3, p. 244]). If $R$ is a right Bézout domain, then $\rho(A)=\rho_{r}(A)=\rho_{c}(A)$ for any $A$ over $R$. A matrix $A \in R^{n \times n}$ is called full if $\rho(A)=n$. Note that $A \in R^{r \times s}$ is a left zero divisor in $R^{r \times s}$ if and only if $\rho_{c}(A)<r$ (see [3, Collorary, p. 245]). A square matrix $A$ over a right Ore domain is not a left zero-divisor if and only if it is a full matrix (see [3, Proposition 5.2]).

The following important result holds for full matrices over FI-ring.

Proposition 1. [3, Theorem 6.4] If $R$ is an FI-ring then $R^{n \times n}$ is a ring with unique factorization of full matrices, i.e. for any full matrix is either an invertible matrix or is a product atoms and any two decompositions a full matrix are isomorphic.

A matrix $A=\left(a_{i j}\right) \in R^{n \times m}$ is called diagonal if $a_{i j}=0$ for all $i \neq j$ and we write it as $\operatorname{diag}\left(a_{11}, \ldots, a_{n n}\right)$. Two matrices $A$ and $B$ over a ring $R$ is equivalent if there exist invertible matrices $P$ and $Q$ over $R$ such that $B=P A Q$. If a matrix $A$ over $R$ is equivalent to a diagonal matrix $D=\left(d_{i i}\right)$ with the property that $d_{i i}$ is a total divisor of $d_{i+1, i+1}$ (i.e. $R d_{i+1, i+1} R \subset$ $\left.d_{i} R \cap R d_{i}\right)$, then we say that $A$ admits a canonical diagonal reduction. A 
ring $R$ over which every matrix admits a canonical diagonal reduction is called an elementary divisor ring.

A ring $R$ is called right (left) Hermite if each matrix $A \in R^{1 \times 2}\left(A \in R^{2 \times 1}\right)$ admits a diagonal reduction. A ring which is right and left Hermite is called a Hermite ring. Moreover, each elementary divisor ring is Hermite, and a right (left) Hermite ring is a right (left) Bézout ring [9, p. 298-299].

A row $\left(a_{1}, \ldots, a_{n}\right) \in R^{n}$ is called unimodular if $a_{1} R+a_{2} R+\cdots+a_{n} R=R$. An unimodular $n$-row $\left(a_{1}, \ldots, a_{n}\right) \in R^{n}$ over a ring $R$ is called reducible if there exist a $(n-1)$-row $\left(b_{1}, \ldots, b_{n-1}\right) \in R^{n-1}$ such that

$$
\left(a_{1}+a_{n} b_{1}, a_{2}+a_{n} b_{2}, \ldots, a_{n-1}+a_{n} b_{n-1}\right) \in R^{n-1}
$$

is unimodular. If $n \in \mathbb{N}$ is the smallest number such that any unimodular $(n+1)$-row is reducible, then $R$ has stable range $n$, where $n \geq 2$. A ring $R$ has stable range 1 if $a R+b R=R$ implies that $(a+b t) R=R$ for some $t \in R$.

Let $R$ be a simple ring. Clearly, $R a R=R$ for each $a \in R \backslash\{0\}$ and there exist $n \in \mathbb{N}$ and $u_{1}, \ldots, u_{n}, v_{1}, \ldots, v_{n} \in R$ such that

$$
u_{1} a v_{1}+u_{2} a v_{2}+\cdots+u_{n} a v_{n}=1 .
$$

If for all $a \in R \backslash\{0\}$, there exists a minimal $n \in \mathbb{N}$ which satisfies (2), then $R$ is called $n$-simple ring.

A ring with identity $R$ is called unit-regular if for every $a \in R$ there is a unit $u \in U(R)$ with $a=a u a$. A von Neumann regular $\operatorname{ring} R$ is unit-regular if and only if $R$ has stable range 1 .

In the sequel we use freely the following results:

Proposition 2. The following conditions hold:

(i) [6, Theorem 3] Each 2-simple unit-regular ring is an elementary divisor ring;

(ii) [8, Proposition 1.8] Each right Bézout domain is a right Ore domain;

(iii) [9, Corollary 2.1.2, p. 56] Each right (left) Hermite ring is a ring of stable range 2 ;

(iv) [9, Clorollary 2.1.5, p. 60] If $R$ is a right Bézout ring of finite stable range $n \in \mathbb{N}$, then each right (left) unimodular row (column) of length $m \geq n+1$ is completive to an element of the subgroup $G E_{m}(R)$ of elementary matrices of the group $G L_{m}(R)$, with the addition of extra columns and rows. 


\section{Proofs}

We start our proof with the following.

Lemma 1. Let $R$ be an n-simple ring. For any $a_{1}, \ldots, a_{n} \in R$ with the property $a_{1} \cdots a_{n} \neq 0$, there exist $u_{1}, \ldots, u_{n}, v_{1}, \ldots, v_{n} \in R$ such that

$$
\sum_{i=1}^{n} u_{i} a_{i} v_{i}=1
$$

Proof. Since $a_{1} \cdots a_{n} \neq 0$ and $R$ is $n$-simple, $\sum_{i=1}^{n} x_{i}\left(a_{1} \cdots a_{n}\right) y_{i}=1$ for some $x_{1}, \ldots, x_{n}, y_{1}, \ldots, y_{n} \in R$ by (2). Put $u_{1}:=x_{1}, u_{2}:=x_{2} a_{2}, \ldots$, $u_{n}:=x_{n} a_{1} \cdots a_{n-1}, \quad v_{1}:=a_{2} \cdots a_{n} y_{1}, v_{2}:=a_{3} \cdots a_{n} y_{2}, \ldots, v_{n}:=y_{n}$. Obviously, (3) is a consequence of the equation $\sum_{i=1}^{n} x_{i} a_{i} y_{i}=1$.

Lemma 2. Let $R$ be a simple elementary divisor ring. For each $a \in R \backslash\{0\}$ there exist $u_{1}, u_{2}, v_{1}, v_{2} \in R$ such that

$$
\text { either } \quad u_{1} a v_{1}+u_{2} a v_{2}=1 \quad \text { or } \quad u_{1}(1-a) v_{1}+u_{2}(1-a) v_{2}=1 .
$$

Proof. If $a \in R \backslash\{0\}$, then $\operatorname{diag}(a, a) \cdot P=Q \cdot \operatorname{diag}(z, b)$ (see the definition of $R$ ), in which $z, b \in R, P=\left(p_{i j}\right), Q=\left(q_{i j}\right) \in G L_{2}(R)$ and $R b R \subseteq z R \cap R z$. Hence

$$
\left(\begin{array}{ll}
a p_{11} & a p_{12} \\
a p_{21} & a p_{22}
\end{array}\right)=\left(\begin{array}{ll}
q_{11} z & q_{12} b \\
q_{21} z & q_{22} b
\end{array}\right) .
$$

Since $R$ is simple, either $z \in U(R) \quad$ or $\quad b=0$.

Consider each case separately.

Case 1. Let $z \in U(R)$. We can assume $z:=1$, so from (44) we have

$$
a p_{11}=q_{11} \quad \text { and } \quad a p_{21}=q_{21} .
$$

Since $\left(\begin{array}{c}q_{11} \\ q_{21}\end{array}\right) \neq 0$ as the first column of $Q \in G L_{2}(R), R=R q_{11}+R q_{21}$. This yields $1=u q_{11}+v q_{21}:=u_{1} a v_{1}+u_{2} a v_{2}$ for some $u, v \in R$ by (5), where $u_{1}:=u, u_{2}:=v, v_{1}:=p_{11}$ and $v_{2}:=p_{21}$.

Case 2. Let $b=0$. Clearly $\left(\begin{array}{l}p_{12} \\ p_{22}\end{array}\right) \neq 0$ as the second column of $P \in$ $G L_{2}(R)$ and $a p_{12}=a p_{22}=0$ by (44), so

$$
p_{12}=(1-a) p_{12} \quad \text { and } \quad p_{22}=(1-a) p_{22} .
$$

As in the previous case, $\quad 1=x p_{12}+y p_{22}:=u_{1}(1-a) v_{1}+u_{2}(1-a) v_{2}$ for some $x, y \in R$ by (6), where $u_{1}:=x, u_{2}:=y, v_{1}:=p_{12}$ and $v_{1}:=p_{22}$. 
Corollary 1. Let $R$ be a simple elementary divisor ring. For each $a \in R \backslash\{0\}$ there exist $L, M \in G L_{2}(R)$ and $b \in R$, such that

$$
L \cdot \operatorname{diag}(a, a) \cdot M=\operatorname{diag}(1, b) .
$$

Moreover, a simple elementary divisor domain is a 2-simple domain.

Proof. According to the proof of Lemma 2 , if $\operatorname{diag}(a, a) \cdot P=Q \cdot \operatorname{diag}(z, 0)$ then $\operatorname{diag}(1-a, 1-a) \cdot T=S \cdot \operatorname{diag}(1, c)$, where $P, Q, T, S \in G L_{2}(R)$ and $c \in R$. Conversely, if $\operatorname{diag}(1-a, 1-a) \cdot S=T \cdot \operatorname{diag}(t, c)$, where $T, S \in G L_{2}(R)$ and $t, c \in R$, then $\operatorname{diag}(a, a) \cdot P=Q \cdot \operatorname{diag}(1, b)$ for some $P, Q \in G L_{2}(R)$.

If $R$ is a simple elementary divisor domain, then for each $a \in R \backslash\{0\}$ there exist $u_{1}, u_{2}, v_{1}, v_{2} \in R$, such that $u_{1} a v_{1}+u_{2} a v_{2}=1$ by Lemma 2 . The case 2 (see the proof of Lemma 2) is impossible for $a \in R \backslash\{0\}$, so

$$
\operatorname{diag}(a, a) \cdot P=Q \cdot \operatorname{diag}(1, b), \quad\left(P, Q \in G L_{2}(R), b \in R\right) .
$$

The concept of an $(n+1)$-simple ring closely linked to the theory of rings of stable range $n$. First consider 2 -simple rings of stable range 1 .

Lemma 3. Let $R$ be a 2-simple ring of stable range 1. For each $a \in R \backslash\{0\}$ there exist $x, y \in R$ such that $a+x a y \in U(R)$.

Proof. Since $R$ is 2-simple, for each $a \in R \backslash\{0\}$ there exist $u_{1}, u_{2}, v_{1}, v_{2} \in R$ such that $u_{1} a v_{1}+u_{2} a v_{2}=1$, so $u_{1} a R+u_{2} a R=R$. The ring $R$ has stable range 1 , so $\left(u_{2} a+u_{1} a t\right) R=R$ for some $t \in R$ and

$$
u_{1} a t+u_{2} a=w_{1} \in U(R) .
$$

Similarly, $u_{1} a R+u_{2} R=R$ and $u_{1} a s+u_{2}=w_{2} \in U(R)$ for some $s \in R$, so $u_{2}=w_{2}-u_{1}$ as. From (17) we obtain

$$
u_{1} a t+w_{2} a-u_{1} a s a=u_{1} a(t-s a)+w_{2} a=w_{1} \in U(R)
$$

and $x a y+a=x \in U(R)$, in which $x:=w_{2}^{-1} u_{1}$ and $y:=t-s a$.

As a consequence of Lemma 3, we have the following.

Lemma 4. Let $R$ be a 2-simple ring of stable range 1. Each matrix $0 \neq$ $\operatorname{diag}(a, a) \in R^{2 \times 2}$ can be reduce to $\operatorname{diag}(1, b) \in R^{2 \times 2}$ for some $b \in R$. 
Proof. Let $a \neq 0$. Since $x a y+a=u \in U(R)$ for some $x, y \in R$ by Lemma 6 ,

$$
\left(\begin{array}{cc}
u^{-1} & 0 \\
0 & 1
\end{array}\right) \cdot\left[\left(\begin{array}{ll}
1 & x \\
0 & 1
\end{array}\right)\left(\begin{array}{ll}
a & 0 \\
0 & a
\end{array}\right)\left(\begin{array}{ll}
1 & 0 \\
y & 1
\end{array}\right)\right]=\left(\begin{array}{cc}
1 & u^{-1} x a \\
a y & a
\end{array}\right)
$$

and $S\left(\begin{array}{cc}1 & u^{-1} x a \\ a y & a\end{array}\right) \cdot T=\operatorname{diag}(1, b)$ for some $b \in R$ and $S, T \in G L_{2}(R)$.

Proof of Theorem 1. For any $a, b \in R$ with $a b \neq 0$ there exist $u_{1}, u_{2}, v_{1}, v_{2} \in$ $R$ such that $u_{1} a v_{1}+u_{2} b v_{2}=1$ by Lemma 1. Hence $u_{1} a R+u_{2} b R=R$ and $\left(u_{2} b+u_{1} a t\right) R=R=\left(u_{2}+u_{1} a s\right) R$ for some $s, t \in R$, because $R$ has stable range 1 . Consequently, $u_{1} a t+u_{2} b=w_{1} \in U(R)$ and $u_{1} a s+u_{2}=w_{2} \in U(R)$. It follows that

$$
w_{1}=u_{1} a t+\left(w_{2}-u_{1} a s\right) b=u_{1} a(t-s b)+w_{2} b \in U(R)
$$

and $x a y+b=w_{2}^{-1} w_{1} \in U(R)$, in which $x:=w_{2}^{-1} u_{1}$ and $y:=t-s b$. Finally,

$$
\left(\begin{array}{cc}
w_{1}^{-1} w_{2} & 0 \\
0 & 1
\end{array}\right) \cdot\left[\left(\begin{array}{ll}
x & 1 \\
1 & 0
\end{array}\right)\left(\begin{array}{ll}
a & 0 \\
0 & b
\end{array}\right)\left(\begin{array}{ll}
y & 1 \\
1 & 0
\end{array}\right)\right]=\left(\begin{array}{cc}
1 & w_{1}^{-1} w_{2} x a \\
a y & a
\end{array}\right)
$$

and $S \cdot\left(\begin{array}{cc}1 & w_{1}^{-1} w_{2} x a \\ a y & a\end{array}\right) \cdot T=\operatorname{diag}(1, c)$ for some $c \in R$ and $S, T \in G L_{2}(R)$.

The case when $a b=0$, but $b a \neq 0$ can be treated similarly.

Lemma 5. A simple unit-regular ring $R$ is an elementary divisor ring if and only if for each idempotent $e \in R$, there exist $u_{1}, u_{2}, v_{1}, v_{2} \in R$ such that

$$
u_{1} e v_{1}+u_{2} e v_{2}=1 \text {. }
$$

Proof. This is a simple consequence of Lemma 2.

Proof of Theorem 2. According to the restrictions imposed on $R$ and $A$ we have $A T=\operatorname{diag}\left(\varepsilon_{1}, \ldots, \varepsilon_{n+1}\right) \neq 0$ for some matrix $T$. Since $R$ is a $(n+1)$ simple domain, we have

$$
u_{1} \varepsilon_{1} v_{1}+u_{2} \varepsilon_{2} v_{2}+\cdots+u_{n+1} \varepsilon_{n+1} v_{n+1}=0
$$

for some $u_{1}, \ldots, u_{n+1}, v_{1}, \ldots, v_{n+1} \in R$. It follows that

$$
\left(u_{1} u_{2}, \ldots, u_{n+1}\right) \cdot A \cdot\left(w_{1} w_{2}, \ldots, w_{n+1}\right)^{T}=1,
$$


in which $\left(w_{1}, \ldots, w_{n+1}\right)^{T}=T^{T} \cdot\left(u_{1}, \ldots, u_{n+1}\right)^{T}$ and where $\left(w_{1}, \ldots, w_{n+1}\right)^{T}$ is the transposed matrix of $\left(w_{1}, \ldots, w_{n+1}\right)$. This yields

$$
u_{1} R+\cdots+u_{n+1} R=R w_{1}+\cdots+R w_{n+1}=R .
$$

Since $R$ has stable range $n$, the row $\left(u_{1}, \ldots, u_{n+1}\right)$ and the column $\left(w_{1}, \ldots, w_{n+1}\right)^{T}$ can be completed (see Proposition 2(iv)) to the following matrices

$$
U=\left(\begin{array}{ccc}
u_{1} & \cdots & u_{n+1} \\
& \star &
\end{array}\right), \quad W=\left(\begin{array}{c}
w_{1} \\
\vdots \\
w_{n+1}
\end{array}\right) \in G E_{n+1}(R) .
$$

Finally, $U A W=\left(\begin{array}{cc}1 & * \cdots * \\ \vdots & B \\ * & \end{array}\right)$ by (8) and using elementary transformations of rows and columns it can be transformed to the form (2).

Proof of Theorem 4. The fact that each $A_{i} \in R^{n \times n}$ is a triangular matrix follows from the fact that each commutative Bézout domain is a Hermite ring see [9, p. 29-30]. 


\section{References}

[1] S. A. Amitsur. Remarks on principal ideal rings. Osaka Math. J., 15:5969, 1963.

[2] P. Ara, K. R. Goodearl, K. C. O’Meara, and E. Pardo. Diagonalization of matrices over regular rings. Linear Algebra Appl., 265:147-163, 1997.

[3] P. Cohn. Svobodnye kol'tsa $i$ ikh svyazi. Izdat. "Mir", Moscow, 1975. Translated from the English by L. A. Bokut'.

[4] P. M. Cohn. Right principal Bezout domains. J. London Math. Soc. (2), 35(2):251-262, 1987.

[5] P. M. Cohn and A. H. Schofield. Two examples of principal ideal domains. Bull. London Math. Soc., 17(1):25-28, 1985.

[6] M. Henriksen. On a class of regular rings that are elementary divisor rings. Arch. Math. (Basel), 24:133-141, 1973.

[7] P. Menal and J. Moncasi. On regular rings with stable range 2. J. Pure Appl. Algebra, 24(1):25-40, 1982.

[8] B. Stenström. Rings and modules of quotients. Lecture Notes in Mathematics, Vol. 237. Springer-Verlag, Berlin-New York, 1971.

[9] B. Zabavsky. Diagonal reduction of matrices over rings, volume 16 of Mathematical Studies Monograph Series. VNTL Publishers, Lviv, 2012. 